\title{
A method for rapid analysis of the root hydrotropic response in Arabidopsis thaliana
}

\author{
L Noriega-Calixto L', María E Campos' \& Gladys I Cassab*,1
}

\begin{abstract}
The system for analyzing the hydrotropic curvature with a moisture gradient in wild-type Arabidopsis roots was modified. Optimal conditions were determined for detecting a hydrotropic curvature of $90^{\circ}$ just after $4 \mathrm{~h}$ of stimulation. This system only requires $15 \mathrm{ml}$ of a solution of $\mathrm{K}_{2} \mathrm{CO}_{3}$ with a density of $1.48 \mathrm{~g} \cdot \mathrm{mll}^{-1}$ to generate a rapid moisture gradient inside a square Petri dish without decreasing root growth. In this, the root growth rate observed in hydrostimulated wild-type and miz 1 mutant, utilized as a negative control, increases sixfold compared with those roots examined using the former method.
\end{abstract}

\section{METHOD SUMMARY}

A modified bioassay was established to examine the root hydrotropic response of Arabidopsis in a square Petri dish containing $15 \mathrm{ml}$ of a solution of $\mathrm{K}_{2} \mathrm{CO}_{3}$ with a density of $1.48 \mathrm{~g} \cdot \mathrm{ml}^{-1}$. The root curvature was rapidly induced in the presence of a moisture gradient and reached $90^{\circ}$ after $4 \mathrm{~h}$. Root elongation and growth rate were maintained in these conditions for $8 \mathrm{~h}$.

\section{KEYWORDS:}

Arabidopsis thaliana $\cdot$ hydrotropic curvature $\cdot$ moisture gradient

'Departamento de Biología Molecular de Plantas, Instituto de Biotecnología, Universidad Nacional Autónoma de México, Av. Universidad 2001, Col. Chamilpa, Cuernavaca, Mor., 62210, México *Author for correspondence: gladys@ibt. unam.mx

BioTechniques 66: 00-00 (March 2019) 10.2144/btn-2018-0173

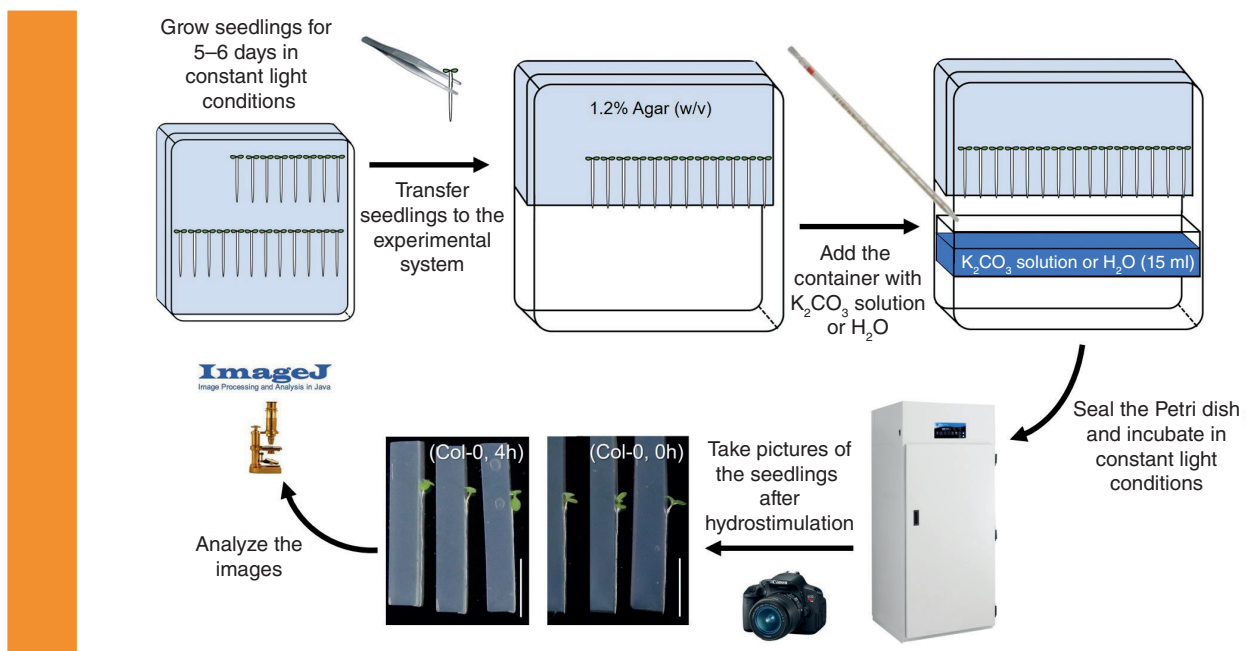

\section{INTRODUCTION}

In view of the increasing demand for food by growing populations and of the limited water availability in many countries due to global warming, there is an urgent need to understand what root properties facilitate the extraction of water from drying soils [1]. Global warming threatens many aspects of human life [2] by reducing agricultural harvests, resulting in widespread risk of food insecurity and social problems [3-6]. Climate change has already altered the distribution of species and phenotypic variation, and the impact of changing climates is expected to intensify since there are very few attempts to stop $\mathrm{CO}_{2}$ emissions [7]. By 2040, droughts and other extreme weather will be commonplace $[8,9]$, hence improving tolerance to drought and heat is a foremost target of crop improvement programs. However, tolerance can come with trade-offs; for example, it has long been known that stress-tolerant plants have lower growth rates and productivity [10]. Plants have evolved a variety of mechanisms to adapt to drought conditions [11]. In recent decades, researchers have identified a number of traits that might help plant roots to acquire water efficiently and tolerate desiccation, as well as a smaller number of root traits that may assist soil water acquisition [12]. Importantly, when roots encounter changes in environmental conditions they will change growth direction in order to optimize plant survival. Such directional changes in response to several stimuli (tropisms) include roots sensing soil water content and growing towards water to avoid dry soil, by either changing direction or halting growth. Hydrotropism is a physiological trait that most probably evolved to avoid drought [13-15]. Root growth direction is strongly directed by gravity and also by other stimuli such as light, obstacles and nutrients. However, root gravitropic response influences all the other root tropic responses, since gravity is ubiquitous in Earth. This makes it problematic to observe hydrotropism in a short period of time since the early tropic response of roots is to follow the direction of the gravity vector. Despite water sensing being the subject of very early plant physiology studies, until recently the mechanisms of this growth response were essentially unknown. Phenotyping and screening procedures in model plant and crop species are needed for genetic dissection of complex biological processes such as hydrotropism $[16,17]$. Therefore, $\bullet$ 
- an easier protocol that allows the analysis of the hydrotropic response in Arabidopsis seedlings would help to isolate new genes implicated in this response. Takahashi et al. [18] and Eapen et al. [19] described two different protocols to assess the hydrotropic response in root of Arabidopsis seedlings. The basis of both protocols is the stimulation of roots with water potential or moisture gradients, with it expected that it will cause root curvature toward the water source and not to the gravity vector. The utilization of these protocols has allowed the isolation of $\mathrm{Arabi}$ dopsis mutants that show an absence of a normal hydrotropic response such as miz1, miz2/gnom, ahr1 and nhr1 [19-22]. Some genes required for hydrotropism, such as MIZ1 and MIZ2/GNOM [20,21] have been thoroughly characterized and it has been demonstrated that MIZ1 participates in the generation of a systemic $\mathrm{Ca}^{2+}$ signal by its direct binding to and inhibition of ECA1, an endoplasmic reticulum $\mathrm{Ca}^{2+}$ pump, which is essential for water tracking [22]. Moreover, the site of water perception and differential growth control was localized to the root cortex [23]. On the other hand, some plant growth regulators such as $A B A$, cytokinin and brassinosteroids have been implicated in hydrotropism regulation [14,24-28]. In particular, ABA signaling through PYR/PYL/RCAR receptors is required for hydrotropism in Arabidopsis [26]. Recently, it was reported that the apoplastic proton extrusion in root tips is influenced by brassinosteroids (BRs) receptor BR-INSENSITIVE1 (BRI1), which is also crucial for the hydrotropic root response in Arabidopsis [27,28]. Whereas loss-of-function mutations in the ubiquitously expressed BRI1 receptor leads to drought resistance at the expense of growth, overexpression of the BRL3 receptor confers drought tolerance without penalizing overall growth [29]. Hence, BRs signaling links the hydrotropic root response with drought resistance. In addition, it has been previously suggested that the genes involved in hydrotropism could be important targets for crop improvement by enhancing drought avoidance $[13,30]$. A recent demonstration that a robust hydrotropic response leads to better growth under drought and partial lateral irrigation in different maize cultivars strongly supports this notion [31]. Here, we describe a system for examining the root hydrotropic response with a moisture gradient in Arabidopsis. The previous system that utilized moisture gradients for inducing a hydrotropic curvature in Arabidopsis reported by Kobayashi et al. [20] was modified for optimizing the conditions for detecting the curvature of $90^{\circ}$ just after $4 \mathrm{~h}$ of stimulation. Moreover, our system requires only $15 \mathrm{ml}$ of $\mathrm{K}_{2} \mathrm{CO}_{3}$ solution with a density of $1.48 \mathrm{~g} \cdot \mathrm{ml}^{-1}$ to generate the moisture gradient, compared with the $500 \mathrm{ml}$ described earlier. Finally, root growth of Arabidopsis seedlings increased approximately sixfold compared with the aforementioned system.

We modified the system reported by Takahashi et al. [18] and determined the optimal conditions for detecting the hydrotropic curvature in Arabidopsis in just $4 \mathrm{~h}$ (Figure 1A\&B). We first analyzed the effect of exposing seedling roots of wild-type (Col-0) and miz1 mutants to two $\mathrm{K}_{2} \mathrm{CO}_{3}$ solutions

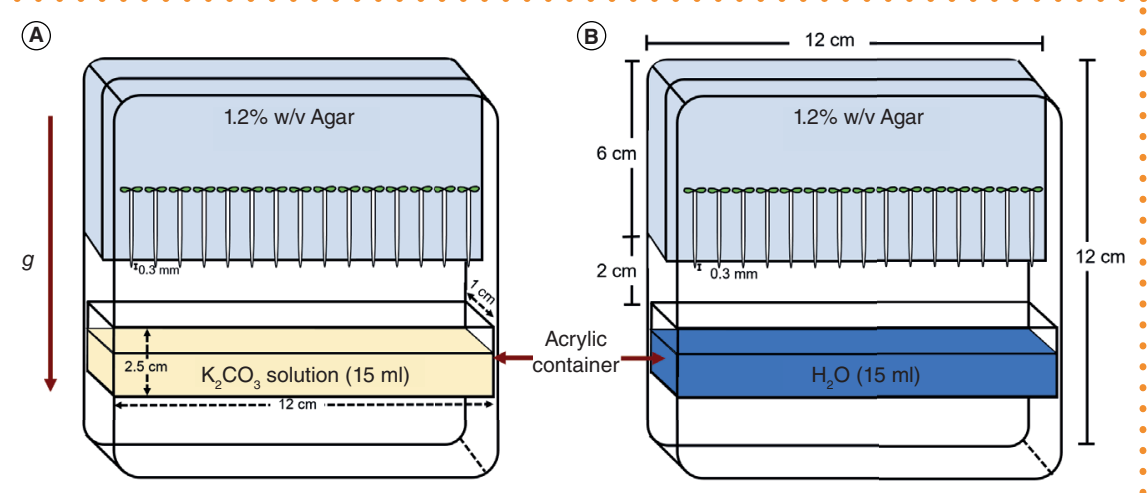

Figure 1. Schematic representation of the experimental system to analyze the root hydrotropic response of Arabidopsis. (A) Experimental system containing the solution of $\mathrm{K}_{2} \mathrm{CO}_{3}$ with a density of $1.48 \mathrm{~g} \cdot \mathrm{ml}^{-1}$. (B) Control system containing $\mathrm{H}_{2} \mathrm{O}$.

with different densities, $0.74 \mathrm{~g} \cdot \mathrm{ml}^{-1}$ and $1.48 \mathrm{~g} \cdot \mathrm{ml}^{-1}$, respectively, and $\mathrm{H}_{2} \mathrm{O}$ as a control condition. Wild-type and miz 1 seedling roots exposed for $12 \mathrm{~h}$ to the $\mathrm{K}_{2} \mathrm{CO}_{3}$ solution with a density of $0.74 \mathrm{~g} \cdot \mathrm{ml}^{-1}$ showed vertical growth following the direction of the gravity vector as did those subjected to $\mathrm{H}_{2} \mathrm{O}$ (data not shown). By contrast, roots of wild-type seedlings exposed to the $\mathrm{K}_{2} \mathrm{CO}_{3}$ solution with a density of $1.48 \mathrm{~g} \cdot \mathrm{ml}^{-1}$ developed a hydrotropic curvature toward the $\mathrm{H}_{2} \mathrm{O}$ source (agar block), while roots of the miz1 mutant displayed their characteristic absence of hydrotropic response [20]. Hence, we utilized the $\mathrm{K}_{2} \mathrm{CO}_{3}$ solution with a density of $1.48 \mathrm{~g} \cdot \mathrm{ml}^{-1}$ for our further analyses.

We measured the relative humidity (RH) at three different positions in the square Petri dish $+\mathrm{K}_{2} \mathrm{CO}_{3}$ solution (Figure $2 \mathrm{~A} \& \mathrm{~B}$ ). Measurements of $\mathrm{RH}$ were also made in the control system $\left(+\mathrm{H}_{2} \mathrm{O}\right.$ ) (Figure $2 \mathrm{C}$ ). After $1 \mathrm{~h}$ of sealing the plate of the control system, the $\mathrm{RH}$ at the three different positions was close to $90 \%$, which was nearly sustained for $23 \mathrm{~h}$. Thus, no substantial moisture gradient was observed under this condition (Figure 2C). However, the $\mathrm{RH}$ in the experimental system $+\mathrm{K}_{2} \mathrm{CO}_{3}$ solution decreased to $83 \%$ in the lower and central part of the plate, differing to the $\mathrm{RH}$ of $89 \%$ detected in the upper part (Figure 2B). Afterwards, the $\mathrm{RH}$ measured in the lower and central part of the plate showed a very mild increase for the next $4 \mathrm{~h}$, reaching about $88 \%$ after $23 \mathrm{~h}$. The $\mathrm{RH}$ at the upper part of the plate of the experimental system $+\mathrm{K}_{2} \mathrm{CO}_{3}$ solution was around $88 \%$ at all time points examined (Figure $2 \mathrm{~B}$ ).

Once the conditions for the development of a moisture gradient were established in the experimental system $+\mathrm{K}_{2} \mathrm{CO}_{3}$ solution, we analyzed the hydrotropic root curvature at 3,4 and $8 \mathrm{~h}$ after hydrostimulation (Figures $3 \& 4$ ). Roots of wild-type seedlings showed a hydrotropic curvature of nearly $76^{\circ}$ after merely $3 \mathrm{~h}$ of stimulation, while roots of the miz1 mutant did not display a hydrotropic response (Figures $3 \& 4 A$ ). After $4 \mathrm{~h}$, roots of the wild-type developed a curvature of $90^{\circ}$ (Figures $3 \& 4 \mathrm{~A}$ ). The rapid development of the hydrotropic curvature up to $90^{\circ}$ in wild-type roots might be related to the formation of a moisture gradient in $1 \mathrm{~h}$ close to their tips $(2 \mathrm{~cm})$ (Figure 2A \& B). In the experimental system $+\mathrm{K}_{2} \mathrm{CO}_{3}$ solution, root elongation and growth rate of both wild-type and miz1 roots was equivalent 


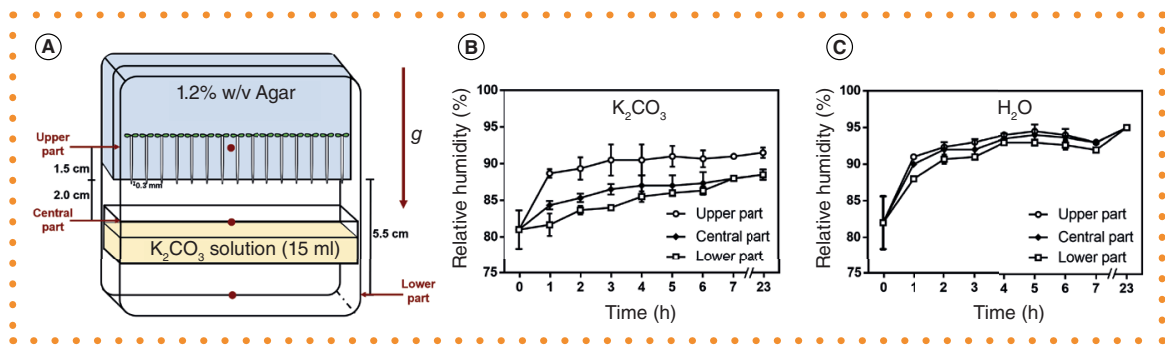

Figure 2. Generation of a moisture gradient in the experimental system. (A) The diagram indicates the places in the plate where the relative humidity $(\mathrm{RH})$ was measured. The $\mathrm{RH}$ was measured at $1.5 \mathrm{~cm}$ above (upper part, open circles), $2.0 \mathrm{~cm}$ (central part, filled diamonds) and $5.5 \mathrm{~cm}$ (lower part, open squares) below the agar edge placed in the square Petri dish containing $\mathrm{K}_{2} \mathrm{CO}_{3}$ solution $\left(1.48 \mathrm{~g} \cdot \mathrm{ml}^{-1}\right)$ or $\mathrm{H}_{2} \mathrm{O}$. (B \& C) Graphs indicate the RH detected in the experimental system plus the $\mathrm{K}_{2} \mathrm{CO}_{3}$ solution (B) and in the control system plus $\mathrm{H}_{2} \mathrm{O}(\mathrm{C})$ at $0,1,2,3,4,5,6,7$ and $23 \mathrm{~h}$ after sealing the Petri dish. Each measurement was repeated three times for every time point in a time-course experiment.

- after 3 and $4 \mathrm{~h}$ of stimulation (Figure $4 \mathrm{~B}$ ). The growth rate of wild-type and miz1 roots was nearly $0.35 \mathrm{~mm} \cdot \mathrm{h}^{-1}$ at these time points (Figure 4C). Thus, the conditions of the experimental system $+\mathrm{K}_{2} \mathrm{CO}_{3}$ solution did not impair growth in both wild-type and miz1 seedling roots for $4 \mathrm{~h}$ (Figure $4 \mathrm{C}$ ). However, growth rate of miz1 roots decreased after $8 \mathrm{~h}$ compared with the wild-type (Figure 4C).
In the absence of moisture gradient, no hydrotropic curvature was elicited in either wild-type or miz1 roots (Figures 3 \& 4D). In the control system, growth rate of the wild-type roots was comparable to those of the mizl mutant (Figure 4F).

Takahashi et al. [18] and Eapen et al. [19] designed experimental systems for the induction of the hydrotropic response in

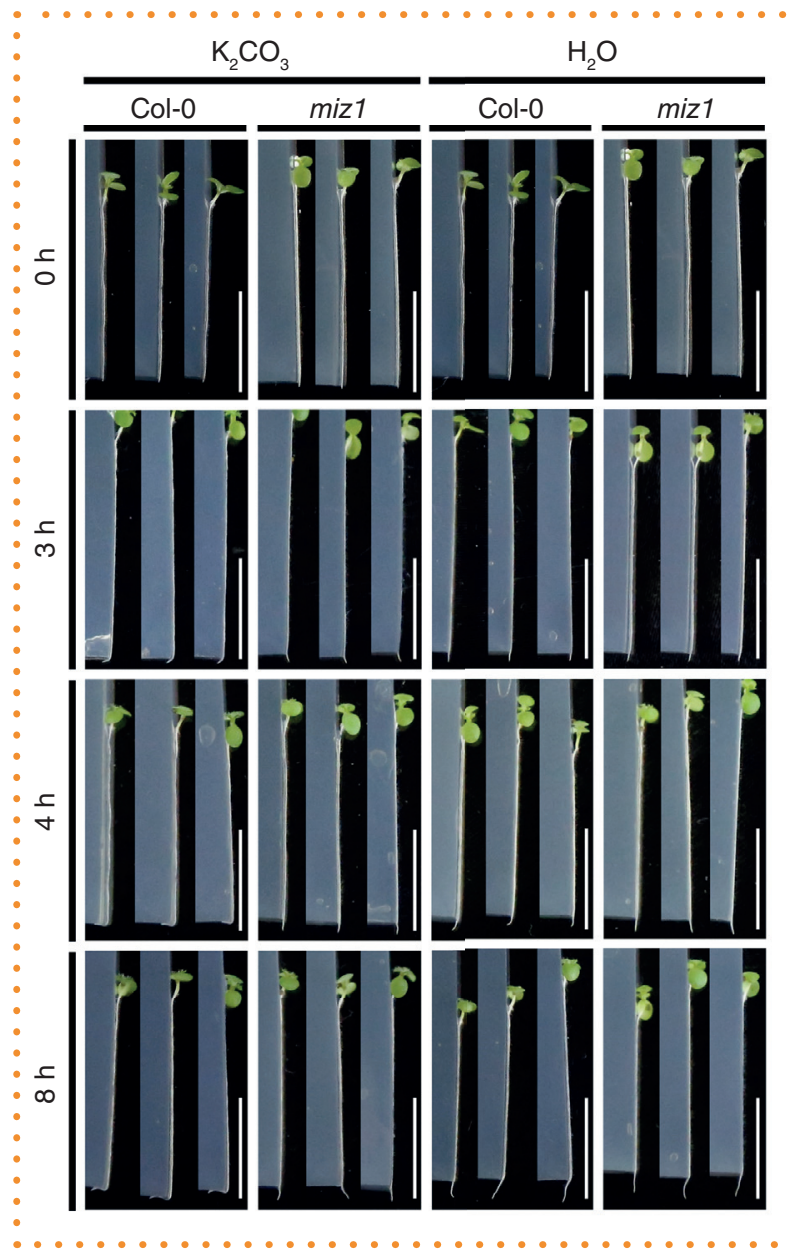

Figure 3. Root hydrotropic curvature of wild-type (Col-0) and miz 1 mutant seedlings in the experimental system containing the $\mathrm{K}_{2} \mathrm{CO}_{3}$ solution or $\mathrm{H}_{2} \mathrm{O}$. Three representative images show roots of Col-0 and miz1 mutant seedlings at $0,3,4$ and $8 \mathrm{~h}$ after the start of hydrotropic stimulation with $\mathrm{K}_{2} \mathrm{CO}_{3}$ solution. The roots of wild-type (Col-0) and mizl mutant seedlings showed an orthogravitropic growth in the control system containing $\mathrm{H}_{2} \mathrm{O}$ at $0,3,4$ and $8 \mathrm{~h}$ after sealing the square Petri dish. Bar $=1 \mathrm{~cm}$.
Arabidopsis roots. These systems generated a water potential gradient in agar by including an osmolyte (sorbitol or glycerol) in square Petri plates. Antoni et al. [17] and Shkolnik et al. [22] modified the split-agar/ sorbitol system of Takahashi et al. [18] by placing Arabidopsis root tips closer to the border between the two media, and the method requires at least $6 \mathrm{~h}$ for observing a hydrotropic curvature of $40^{\circ}$ [26]. Takahashi et al. [18] and Kobayashi et al. [20] described another experimental system that generated a moisture gradient with a saturated solution of $\mathrm{K}_{2} \mathrm{CO}_{3}$ in an acrylic box in which Arabidopsis roots developed a curvature close to $90^{\circ}$ after $8 \mathrm{~h}$ of stimulation.

In the previous experimental system reported by Takahashi et al. [18] and Kobayashi et al. [20], the authors used 4-day-old seedlings for analyzing the hydrotropic response. After $4 \mathrm{~h}$ of stimulation, the authors reported that wild-type seedling roots showed a hydrotropic curvature near $50^{\circ}$, and the root growth rate of wild-type and miz1 mutant seedlings was $0.05 \mathrm{~mm} \cdot \mathrm{h}^{-1}$. In our experimental system, we used 5-6-day-old seedlings and the wild-type roots reached a hydrotropic curvature of $90^{\circ}$ after $4 \mathrm{~h}$ of hydrostimulation displaying a root growth rate of $0.3 \mathrm{~mm} \cdot \mathrm{h}^{-1}$, which was similar to those observed in miz1 mutant roots. Therefore, in this system wild-type roots showed an increase of sixfold in their growth rate compared with those roots incubated in the previous system.

The changes observed between these two experimental systems might be caused by the difference in the age of the seedlings, the close contact of root tips to a moisture gradient generated after $1 \mathrm{~h}$ of sealing the plate, and also by the fact that we did not use a saturated solution of $\mathrm{K}_{2} \mathrm{CO}_{3}$. Previous analysis of hydrotropism in maize demonstrated that a solution of $\mathrm{K}_{2} \mathrm{CO}_{3}$ with a density of $1.48 \mathrm{~g} \cdot \mathrm{ml}^{-1}$ did not cause dehydration of roots after $24 \mathrm{~h}$ of the start of hydrotropic stimulation [28]. In our experimental system, the root growth rate of the miz 1 mutant seedlings decreased after $8 \mathrm{~h}$ of the hydrostimulation, as reported by Kobayashi [20] (Figure 4B \& C). This decrease might be related to the fact that miz1-mutant roots did not bend to the agar block and were exposed longer to the low $\mathrm{RH}$ conditions generated in the experimental system. 

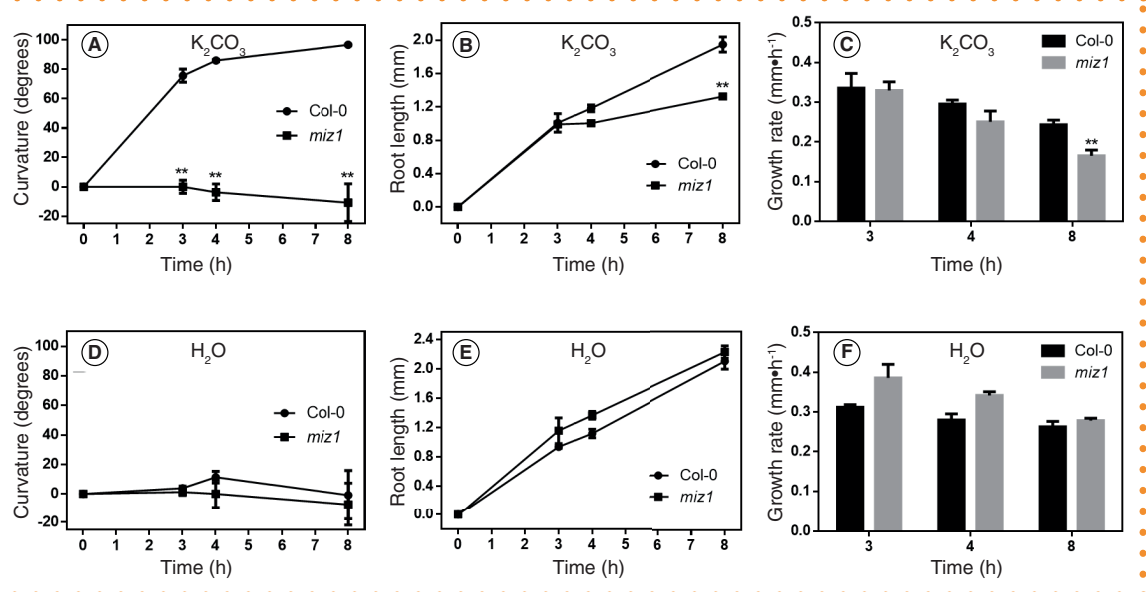

Figure 4. Time course of root hydrotropic curvature, elongation and growth rate of wild-type and miz1 mutant seedlings. The root curvature $(A)$ and the increment of root length (B) were measured at $0,3,4$, and $8 \mathrm{~h}$ after the start of hydrotropic stimulation. The root growth rate (C) was calculated by dividing root length $(\mathrm{mm})$ by time $(\mathrm{h})$. Seedling roots of the miz1 mutant were used as negative control. In the control system, the lack of root hydrotropic response of both Col-0 and miz1 mutant is shown in (D), as well as their elongation (E) and growth rate (F). Each data point is the average of measurements of $\mathbf{3 0}$ individuals from four independent experiments. Error bars represent SD. Asterisks indicate statistically significant differences in the means as determined by Student's t-test.

$* * \mathrm{p}=0.01$.

Filled circles, wild-type Col-0; filled squares, miz1 mutant seedlings.

- In summary, we developed a simple and low-cost experimental system for analyzing hydrotropism in Arabidopsis roots, in which roots developed a hydrotropic curvature of $90^{\circ}$ in just $4 \mathrm{~h}$ after the start of the hydrostimulation. Likewise, this experimental system required only agar and a small volume of $\mathrm{K}_{2} \mathrm{CO}_{3}$ solution within a square Petri plate. We propose that this system represents a useful tool for identifying new elements implicated in the early signaling of the hydrotropic response and for isolating new hydrotropic mutants.

\section{AUTHOR CONTRIBUTIONS}

LNC and MEC designed the experimental system and performed the experiments. LNC and GIC wrote the manuscript.

\section{FINANCIAL \& COMPETING INTERESTS DISCLOSURE}

This work was supported by two grants from the Consejo Nacional de Ciencia de Tecnología (CONACYT), México CB \# 177107 and PN \# 247732. LNC was supported by a fellowship from CONACYT \# 303753 and by an additional fellowship from CONACYT PN \# 247732. The authors have no other relevant affiliations or financial involvement with any organization or entity with a financial interest in or financial conflict with the subject matter or materials discussed in the manuscript apart from those disclosed.

No writing assistance was utilized in the production of this manuscript.

\section{ACKNOWLEDGMENTS}

We are very grateful to Dr Hideyuki Takahashi, Tohoku University, Sendai, Japan, for seed donation of the miz1 mutant. We sincerely thank Delfeena Eapen, Jesús MartínezGuadarrama, y Citlali Benítez for sharing their data of the two densities of the $\mathrm{K}_{2} \mathrm{CO}_{3}$ solution used in this work. We also acknowledge Roberto Rodríguez-Bahena and Shirley Ainsworth for excellent computer and bibliography assistance.

\section{OPEN ACCESS}

This work is licensed under the AttributionNonCommercial-NoDerivatives 4.0 Unported License. To view a copy of this license, visit http://creativecommons.org/licenses/ by-nc-nd/4.0/

\section{SUPPLEMENTARY DATA}

A supplementary protocol accompanies this paper. Reference 32 is in the supplementary data. To view the supplementary data that accompany this paper please visit the journal website at: www.future-science. com/doi/suppl/10.2144/btn-2018-0173

\section{REFERENCES}

1. Battisti DS, Naylor RL. Historical warnings of future food insecurity with unprecedented seasonal heat. Science 323(5911), 240-244 (2009).

2. Bohra-Mishra P, Oppenheimer M, Hsiang SM. Nonlinear permanent migration response to climatic variations but minimal response to disasters. Proc. Natl Acad. Sci. USA 111(27), 9780-9785 (2014).

3. Peng S, Huang J, Sheehy JE et al. Rice yields decline with higher night temperature from global warming. Pro (2004).

4. Takeda S, Matsuoka M. Genetic approaches to crop improvement: responding to environmental and population changes. Nat. Rev. Genet. 9(6), 444-457 (2008).

5. Godfray HC, Beddington JR, Crute IR et al. Food security. the challenge of feeding 9 billion people. Science 327(5967), 812-818 (2010).

6. Mba C, Guimaraes EP, Ghosh K. Re-orienting crop 21 st Century. Agricul. Food Sec. 1(1), 7 (2012).

7. Lobell DB, Schlenker W, Costa-Roberts J. Climate trends and global crop production since 1980 . Science 333 616-620 (2011)

8. Steffen W, Rockströma J, Richardson K et al. Trajectories of the Earth system in the anthropocene. Proc. Nat/ Acad. Sci. USA 115(33), 8252-8259 (2018).

9. Turner WR. Natural climate solutions. Nat. Clim. Change 8, 18-19 (2018)

10. liifs $C$. Integrated responses of plants to stress: a centralized system of physiological responses. BioScience 41, 29-36 (1991).

11. Cattivelli L, Rizza F, Badeck FW et al. Drought tolerance improvement in crop plants: an integrated view from breeding to genomics. Field Crops Res. 105(1-2), 1-14 (2008).

12. Lynch JP. Steep, cheap and deep: an ideotype to optimize water and $\mathrm{N}$ acquisition by maize root systems. Ann. Bot.112(2), 347-357 (2013)

13. Cassab GI, Eapen D, Campos ME. Root hydrotropism: an update. Am. J. Bot. 100(1), 14-24 (2013).

14. Moriwaki T, Miyazawa Y, Kobayashi A, Takahashi H. Molecular mechanism of hydrotropism in seedling roots of Arabidopsis thaliana (Brassicaceae). Am. J Bot. 100(1), 25-34 (2013).

15. Dietrich D. Hydrotropism: how roots search for water. Exp. Bot. 69, 2759-2771 (2018).

16. Eapen D, Martínez-Guadarrama J, Cassab GI. Assays for root hydrotropism and response to water stress. In: Plant Gravitropism Methods and Protocols. Blancaflor E (Ed.). Springer, NY, USA, 133-142 (2015).

17. Antoni R, Dietrich D, Bennett MJ, Rodríguez PL. Hydrotropism: analysis of the root response to a moisture gradient. In: Environmental Responses in Plants: Methods Duque P (Ed.). Springer Science Business Media, NY, USA, 3-9 (2016).

18. Takahashi N, Goto N, Okada K, Takahashi H. Hydrotropism in abscisic acid, wavy, and gravitropic 16(2), 203-211

19. Eapen D, Barroso ML, Campos M et al. A no hydrotropic response (nhr1) root mutant that responds positively to gravitropism in Arabidopsis. Plant Physiol. 131(2), 536-546 (2003)

20. Kobayashi A, Takahashi A, Kakimoto $Y$ et al A gene essential for hydrotropism in roots. Proc. Natl Acad. Sci. USA 104(11), 4724-4729 (2007).

21. Miyazawa Y, Takahashi A, Kobayahi A, Kaneyasu T, Fujii N, Takahashi $\mathrm{H}$. The GNOM-mediated vesicular Arabidopsis roots. Plant Physiol. 149(2), 835-840 (2009).

22. Shkolnik D, Nuriel R, Bonza MC, Costa A, Fromm H. MIZ1 regulates ECA1 to generate a slow, long-distance phloem-transmitted $\mathrm{Ca}^{2+}$ signal essential for root water tracking in Arabidopsis. Proc. Natl Acad. Sci. USA 115(31) 8031-8036 (2018)

23. Dietrich D, Pang L, Kobayashi A et al. Root hydrotropism is controlled via a cortex-specific growth mechanism. Nat. Plants 3(6), 17057 (2017).

24. Saucedo M, Ponce G, Campos ME et al. An altered hydrotropic response (ahr1) mutant of Arabidopsis recovers root hydrotropism with cytokinin. $J$. Exp. Bot. 63(10), 3587-3601 (2012).

25. Moriwaki T, Miyazawa Y, Fujii N, Takahashi H. Light and abscisic acid signalling are integrated by MIZ1 gene expression and regulate hydrotropic response in roots of Arabidopsis thaliana. Plant Cell Environ. 35(8), 1359-1368 (2012)

26. Antoni R, Gonzalez-Guzman $M$, Rodriguez L et al. Pyrabactin resistance1-like8 plays an important role 
for the regulation of abscisic acid signaling in root. Plant Physiol. 161(2), 931-941 (2013).

27. Miao R, Wang M, Yuan W et al. Comparative analysis of Arabidopsis ecotypes reveals a role for brassinosteroids (2018).

28. Yuan W, Li Y, Li L et al. BR-insensitive1 regulates hydrotropic response by interacting with plasma membrane $\mathrm{H}^{+}$-ATPases in Arabidopsis. Plant Signal. Behav. 13(8), e1486147 (2018)

29. Fàbregas N, Lozano-Elena F, Blasco-Escámez D et al. Overexpression of the vascular brassinosteroid recepto BRL3 confers drought resistance without penalizing plant growth. Nat. Commun. 9(1), 4680 (2018).

30. Eapen D, Barroso ML, Ponce G, Campos ME, Cassab GI. Hydrotropism: root growth responses to water. Trends Hydrotropism: root growth respon.
Plant Sci. 10(1), 44-50 (2005).

31. Eapen D, Martínez-Guadarrama J, Hernández-Bruno 0 , Flores L, Nieto-Sotelo J, Cassab GI. Synergy between root hydrotropic response and root biomass in maize (Zea mays L.) enhances drought avoidance. Plant Sci. 265, 87-99 (2017)

32. Murashigue T, Skoog F. A revised medium for rapid growth and bio assays with tobacco tissue cultures. Physiol. Plant. 15(3), 473-497 (1962). 